\title{
Clima organizacional y Síndrome de Burnout en el profesional de enfermería del Hospital de Jaén
}

\section{Organizacional climate and burnout syndrome in the nursing professional of the Hospital of Jaén}

\author{
Ruth Elizabeth Melendez Santillan', Sonia Tejada Muñoz ${ }^{2}$
}

\section{RESUMEN}

El objetivo de la investigación fue determinar la relación que existe entre el clima organizacional y el síndrome de burnout en el profesional de enfermería del Hospital de Jaén, 2018. La muestra estuvo conformada por 60 profesionales de enfermería, para la recolección de los datos se utilizó el cuestionario Inventory del Síndrome de Burnout de Maslach para la variable síndrome de burnout, con una validez de VC=7.89 > VT: 1.46 y una confiabilidad de 0.89 y para la variable clima organizacional se tomó en cuenta la Escala multidimensional de clima organizacional (EMCO), con un nivel de confiabilidad $>0.70$ y validez de $\mathrm{VC}=0.80$ y VT=1.25. Los resultados demuestran que un 46.7\% (28) presenta síndrome de burnout de niel alto, con clima organizacional regular, el 35\% (21) presenta un síndrome de burnout medio con clima Organizacional regular y el 6.7\% (4) tiene síndrome de burnout de nivel bajo con un clima organizacional de nivel malo, concluyéndose así que existe relación altamente significativa entre el síndrome de burnout y clima organizacional $(\mathrm{X} 2 \mathrm{c}=10.684>\mathrm{X} 2 \mathrm{t}=$ 9.4877, $\mathrm{GL}=4, \mathrm{p}=0,030<\alpha=0.05$ ) en esta población en estudio.

Palabras claves: síndrome de burnout, clima organizacional, profesional de enfermería

\begin{abstract}
The objective of the research was to determine the relationship between the organizational climate and the burnout syndrome in the nursing professional of the Hospital de Jaén, 2018. The sample was made up of 60 nursing professionals, for the data collection was used The Maslach Burnout Syndrome Inventory questionnaire for the burnout syndrome variable, with a validity of $\mathrm{VC}=7.89>\mathrm{VT}$ : 1.46 and a reliability of 0.89 and for the organizational climate variable, the Multidimensional Scale of Organizational Climate (EMCO ), with a level of reliability $>0.70$ and validity of $\mathrm{VC}=0.80$ and $\mathrm{VT}=1.25$. The results show that $46.7 \%$ (28) present high-level burnout syndrome, with a regular organizational climate, $35 \%$ (21) present a medium burnout syndrome with a regular organizational climate, and 6.7\% (4) have burnout syndrome. low level with a bad organizational climate, thus concluding that there is a highly significant relationship between burnout syndrome and organizational climate $(\mathrm{X} 2 \mathrm{c}=10.684>\mathrm{X} 2 \mathrm{t}=9.4877, \mathrm{GL}=4, \mathrm{p}=0.030<\alpha=0.05)$ in this study population.
\end{abstract}

Keywords: burnout syndrome, organizational climate, nursing professional

\footnotetext{
Licenciada en enfermería - Facultad de Ciencias de la Salud de la Universidad Nacional Toribio Rodríguez de Mendoza de Amazonas. Email: Mlendezsantillan@gmail.com

${ }^{2}$ Doctora en Ciencias de Enfermería, docente nombrado en categoría de asociado a tiempo completo de la Facultad de Ciencias de la Salud de la Universidad Nacional Toribio Rodríguez de Mendoza de Amazonas. Email: cieloceleste120@hotmail.com
} 


\section{INTRODUCCIÓN}

A nivel mundial el síndrome de Burnout se le ha denominado como un estrés laboral, que tiene tres componentes que lo definen, la desmotivación, el desinterés, el malestar interno o la insatisfacción laboral (Maslach y Jackson, 1981), también se agrupan en tres categorías cansancio emocional, el cual hace referencia al sentimiento de agotamiento por lo que se evidencia desinterés por el trabajo, la despersonalización caracterizada por el rechazo hacia los pacientes y la falta de realización personal que se percibe como la actitud negativa hacia uno mismo, el trabajo, pérdida de interés, irritabilidad, baja productividad y baja autoestima (Gil monte, 2014, p.15)

El Burnout es un síndrome frecuente entre profesionales que realizan actividades de ayuda a otras personas, siendo la enfermería una de las profesiones que más lo padece, a la vez que se perfila como uno de los motivos importantes de abandono de la profesión (Torres, et al, 2015, p.1).

El clima organizacional y el síndrome de burnout, son componentes en el comportamiento cotidiano de los profesionales de salud como consecuencia parte importante del proceso de modernización de una institución, los hospitales son organizaciones públicas donde laboran personas dentro de un espacio delimitado atendiendo personas que en su mayoría presentan problemas de salud, etc. En esas condiciones de trabajo alrededor del $60 \%$ manifiestan el exceso por carga de trabajo y un $19 \%$ falta de apoyo social (Soliz, et al 2015, p.33)

El síndrome de burnout, también conocido como síndrome de desgaste profesional, de sobrecarga emocional o de fatiga en el trabajo, fue declarado en el año 2000 por la Organización Mundial de la Salud (OMS) como un factor de riesgo laboral, en el año 2019 la Organización mundial de la salud ha considerado el reconocimiento oficial del burnout o "síndrome de estar quemado" o de desgaste profesional como enfermedad, tras la ratificación de la revisión número 11 de la clasificación estadística internacional de enfermedades y problemas de salud conexos (CIE-11), aprobada en el año 2018 y cuya entrada en vigencia fijado para el próximo 1 de enero de 2022 (Organización Mundial de la Salud, 2019).

En Colombia se ha reportado diferentes prevalencias de Síndrome de Burnout en profesionales de enfermería que laboran en el área de atención hospitalaria: $15,5 \%$ en Barranquilla, $26,6 \%$ en Cartagena, $24,4 \%$ en Ibagué, $17,5 \%$ y en Orinoquia el $25.5 \%$ referente a los que laboran en otras áreas (Gutiérrez, et al, 2017).
El síndrome de burnout se refiere a una situación que es cada vez más frecuente entre los profesionales de enfermería que presta sus servicios a través de una relación directa y mantenida con las personas, ha sido conceptualizado como una respuesta al estrés laboral crónico y como un trastorno tridimensional de agotamiento emocional, despersonalización y baja realización personal en el trabajo, tiene gran incidencia en profesionales de enfermería a nivel mundial (Acevedo, 2014, p.4).

El 47\% de enfermeras en España sufren síntomas de este síndrome, dicha prevalencia se ha ido incrementando constituyendo un problema social de salud pública, que conlleva a un gran costo económico y social. La exposición prolongada al estrés genera una pérdida de control ante los acontecimientos, de manera que dificulta la práctica de enfermería, disminuyendo la calidad de los cuidados e incrementando el riesgo de que acontezcan efectos no deseados en el paciente y su entorno (Medina, 2015, p.2)

En el hospital de Tercer Nivel de la ciudad de México el profesional de enfermería que labora en áreas críticas presenta un Síndrome de Burnout en niveles significantes relacionados con los turnos de trabajo, con mayor percepción en las dimensiones de cansancio emocional y despersonalización (Rodríguez, 2016, p.19).

En el Perú en el Hospital Central de la Fuerza Aérea, Unidad de Cuidados Críticos se reportó un $15 \%$ de niveles altos en la dimensión despersonalización y un $20 \%$ de nivel alto en la dimensión cansancio emocional en profesionales de enfermería; por otro lado en Arequipa el $21.3 \%$ presenta un nivel alto de agotamiento emocional y baja realización personal y el $28.9 \%$ niveles altos de despersonalización, esto relacionado con el tiempo que lleva laborando en la institución(Arias, 2016,p.12).

Según una investigación realizada en el Hospital Regional Virgen de Fátima de Chachapoyas, en un $66.1 \%$ de enfermeras presentan Síndrome de Burnout, relacionado a factores psicosociales y psicológicos, como la carga mental y sobrecarga laboral (Saucedo, 2015, p.12).

El profesional de enfermería se desempeña dentro de un clima laboral, por ende dentro de un clima organizacional donde la enfermería es considerada una profesión que requiere el despliegue de actividades que involucran control mental y emocional

Como integrantes de un equipo de salud, se caracterizan por el manejo de situaciones críticas con desempeño eficiente y efectivo de ahí que diversos 
autores hayan señalado que esta profesión conlleva a una gran responsabilidad, enfrentándose en forma cotidiana a presiones, conflictos y cambios continuos que conllevarían en el futuro tras varios años de actividad laboral, a ser afectados por el síndrome de burnout (Soliz, et al, 2015, p.33).

El interés por el estudio de clima organizacional ha crecido rápidamente durante los últimos años, ya que las organizaciones a través de la implementación de los sistemas de gestión de calidad la han asumido como uno de los elementos básicos para la detección oportuna de aspectos claves que puedan estar impactando de manera importante el ambiente laboral, de esta forma concebir un mejoramiento continuo mediante la gestión y el desarrollo de nuevas alternativas que permitan el logro de los objetivos de la organización, la motivación mutua, un alto desempeño y el compromiso de la gente en el trabajo, como premisas para elevar la calidad y la productividad (Piñera, et al, 2016).

El desarrollo de perfiles de riesgos del burnout es crucial para avanzar en su prevención, conociendo los factores que influyen en su desarrollo, como la satisfacción o la experiencia profesional, sin embargo, también hay que valorar el papel clave que puede jugar el desarrollo del síndrome de burnout en el servicio hospitalario donde trabajan las enfermeras.

Según lo propuesto y teniendo en cuenta la problemática, se cree que hoy en día los problemas de salud mental está siendo tomado muy a la ligera, sin darnos cuenta que es la base fundamental para el equilibrio de nuestro bienestar físico como mental, en la vida cotidiana, en las relaciones laborales, en el desempeño de nuestro quehacer diario, es por ello es de suma importancia aprender a identificar el estrés laboral, ya sea por sobre demanda de actividades laborales que realizar, relaciones interpersonales negativas, mal manejo de las organizaciones laborales, etc, así mismo diagnosticarlo, tratarlo y de alguna manera dar una alternativa de solución puede ayudar a afrontarle positivamente minimizando sus consecuencias que alteran el estado biopsicosocial y espiritual.

El acceso a los servicios de salud, constituyen un objetivo prioritario para los profesionales y para la organización, orientada necesariamente al paciente dentro de un contexto multidisciplinar.

En este contexto se plantea la siguiente interrogante: ¿Cuál es la relación que existe entre el clima organizacional y el síndrome de burnout en el personal de enfermería del Hospital de Jaén 2018?, cuyo objetivo general fue: determinar la relación que existe entre el clima organizacional y el síndrome de burnout en el profesional de enfermería de este hospital.

\section{MATERIAL Y MÉTODO}

La investigación fue de enfoque cuantitativo, relacional, observacional, prospectivos, transversal (Supo, 2014, p. 1 - 2).

La muestra estuvo conformado por 60 profesionales asistenciales de enfermería del Hospital general de Jaén, 2018, seleccionados de acuerdo a los criterios de inclusión y exclusión por muestreo no probabilístico por conveniencia.

Métodos, técnicas e instrumentos de recolección de datos: para la recolección de los datos en ambas variables, se utilizó el método de la encuesta y técnica del cuestionario (Hernández, et al, 2010).

Para la variable del Síndrome de Burnout: Inventario del síndrome de burnout de Maslach, constituido por 22 ítems en una escala de Likert, y recoge tres dimensiones, cansancio emocional con 9 ítems, despersonalización con 5 ítems y baja realización personal con 8 ítems, con una confiabilidad de 0.90 para aagotamiento emocional, 0.71 para realización personal y 0.79 en despersonalización. Con una validez de $\mathrm{VC}=7.89>$ VT: 1.46 y una confiabilidad de 0.89 (Maslach, C. \& Jackson, S. 1981).

Para la variable clima organizacional: Se tomaron en cuenta escala multidimensional de clima organizacional (EMCO), que se integra de factores estructurados en tres niveles de análisis y se caracteriza por ser una escala de 29 reactivos, con un nivel de confiabilidad $>0.70$ y valide $z$ de $V C=0.80$ y VT=1.25 mediante Alpha de Crombach (Gómez, M. \& Vicario, A. 2008).

\section{RESULTADOS}

Tabla $N^{\circ}$ 01: Relación entre el clima organizacional y el síndrome de burnout en el profesional de enfermería del Hospital de Jaén - 2018.

\begin{tabular}{lcccccccc}
\hline & \multicolumn{9}{c}{ síndrome de burnout } & & \\
\cline { 2 - 7 } \multicolumn{1}{c}{ clima } & \multicolumn{1}{c}{ Alto } & \multicolumn{2}{c}{ Medio } & \multicolumn{3}{c}{ Bajo } & \multicolumn{2}{c}{ TOTAL } \\
\cline { 2 - 8 } organizacional & fi & $\%$ & fi & $\%$ & fi & $\%$ & fi & $\%$ \\
\hline Bueno & 0 & 0.0 & 2 & 3.3 & 1 & 1.7 & 3 & 5.0 \\
Regular & 28 & 46.7 & 21 & 35.0 & 1 & 1.7 & 50 & 83.3 \\
Malo & 4 & 6.7 & 3 & 5.0 & 0 & 0.0 & 7 & 11.7 \\
\hline TOTAL & 32 & 53.3 & 26 & 43.3 & 2 & 3.3 & 60 & 100.0 \\
\hline
\end{tabular}

Tabla $N^{\circ}$ 02: Síndrome de Burnout en el profesional de enfermería del Hospital de Jaén - 2018. 


\begin{tabular}{lcc} 
síndrome de burnout & $\mathbf{f i}$ & $\mathbf{\%}$ \\
\hline Alto & 36 & 60.0 \\
Medio & 22 & 36.7 \\
Bajo & 2 & 3.3 \\
\hline TOTAL & 60 & 100 \\
\hline
\end{tabular}

Tabla $\mathbf{N}^{\circ}$ 03: Clima organizacional en el profesional de enfermería del Hospital de Jaén - 2018.

\begin{tabular}{lcc}
\multicolumn{1}{c}{$\begin{array}{c}\text { clima } \\
\text { organizacional }\end{array}$} & fi & \% \\
\hline Bueno & 3 & 5 \\
Regular & 50 & 83.3 \\
Malo & 7 & 11.7 \\
\hline TOTAL & 60 & 100 \\
\hline
\end{tabular}

Tabla $N^{\circ}$ 04: Síndrome de burnout según dimensiones en el profesional de enfermería del Hospital de Jaén - 2018.

\begin{tabular}{lcccccc}
\hline & \multicolumn{3}{c}{ síndrome de burnout según dimensiones } \\
\cline { 2 - 7 } & $\begin{array}{l}\text { cansancio } \\
\text { emocional }\end{array}$ & despersonalización & \multicolumn{2}{c}{$\begin{array}{c}\text { realización } \\
\text { personal }\end{array}$} \\
\cline { 2 - 7 } categorías & $\mathbf{f i}$ & $\%$ & $\mathbf{f i}$ & $\%$ & $\mathbf{f i}$ & $\%$ \\
\hline Alto & 43 & 71.7 & 42 & 70.0 & 31 & 51.7 \\
Medio & 11 & 18.3 & 15 & 25.0 & 15 & 25.0 \\
Bajo & 6 & 10.0 & 3 & 5.0 & 14 & 23.3 \\
\hline TOTAL & 60 & 100 & 60 & 100 & 60 & 100 \\
\hline
\end{tabular}

Tabla $\mathbf{N}^{\circ}$ 05: Clima organizacional según dimensiones en el profesional de enfermería del Hospital de Jaén - 2018

\begin{tabular}{lcccccc}
\hline & \multicolumn{4}{c}{$\begin{array}{c}\text { clima organizacional según } \\
\text { dimensiones }\end{array}$} \\
\cline { 2 - 7 } categorías & $\begin{array}{c}\text { sistema } \\
\text { individual }\end{array}$ & $\begin{array}{c}\text { sistema } \\
\text { interpersonal }\end{array}$ & \multicolumn{2}{c}{ sistema } \\
& organizacional \\
\cline { 2 - 7 } & $\mathbf{f i}$ & $\mathbf{\%}$ & $\mathbf{F i}$ & $\mathbf{\%}$ & $\mathbf{f i}$ & $\%$ \\
\hline Bueno & 5 & 8.3 & 5 & 8.3 & 3 & 5.0 \\
Regular & 46 & 76.7 & 40 & 66.7 & 34 & 56.7 \\
Malo & 9 & 15.0 & 15 & 25.0 & 23 & 38.3 \\
\hline TOTAL & 60 & 100 & 60 & 100 & 60 & 100 \\
\hline
\end{tabular}

\section{DISCUSIÓN}

El presente estudio ha demostrado que el síndrome de burnout y el clima organizacional tienen una relación altamente significativa $(X 2 \mathrm{c}=10.684>\mathrm{X}$ $2 \mathrm{t}=9.4877, \mathrm{GL}=4, \mathrm{p}=0,030<\alpha=0.05)$.

Se ha encontrado investigaciones como Tipacti, A.
(2016) y Camasi, K. (2018) que tienen similitudes con este estudio cuando afirman que un buen Clima Laboral posee relación directa con el síndrome de burnout.

También se ha podido encontrar que el clima organizacional regular con un síndrome de burnout de nivel alto (Tabla 1), al respecto Chuco, Y. \& Peña, M. (2018) Huancayo encontró que el 38\% presentó un Síndrome de Burnout alto con un mal clima Organizacional malo asimismo el $38 \%$ presenta un síndrome de burnout medio con un regular clima organizacional. Por otro lado, Solís, Z. \& Zamudio, L. y colaboradores (2015) Lima Perú concluyó que el clima organizacional fue nivel regular $81.4 \%$, seguido de un nivel bueno18.6\% y el Síndrome de Burnout corresponde a nivel bajo $86 \%$ y nivel medio $14 \%$.

El profesional de enfermería que presente un cuadro de estrés crónico es consecuencia de un deficiente clima laboral repercutiendo en su salud física mental, como también en su desenvolvimiento y desarrollo del profesional y los directivos de las instituciones públicas y privadas deben tomarle en consideración ya que de ello depende la productividad del recurso humano y por ende la calidad de servicio al usuario.

El síndrome de estar quemado está caracterizado por tres componentes sumamente importantes y de mucho cuidado: el cansancio emocional, la despersonalización y la baja realización personal; considerados en el presente estudio.

Más del 50\% de los profesionales de enfermería que trabajan en el Hospital de Jaén; evidenciaron agotamiento emocional y en menores porcentajes despersonalización y realización personal (Tabla 2).

El Burnout es una respuesta progresiva e insidiosa que evoluciona como parte de un proceso mayor de estrés laboral en profesionales que interactúan directamente con personas (Maslach y Jackson 2006, citado por Miravalles, 2014 p. 2). Este aporte ha sido relevante para medir el síndrome de burnout por niveles, considerándose categorías de medio y bajo como indicios de burnout y el nivel alto a la enfermedad en sí.

Al respecto Cañadas, A. \& García, L. y colaboradores (2016) en España encontró un 44,1\% niveles altos de síndrome de burnout en profesionales de enfermería que realizan jornada física complementaria frente a un $38 \%$ que no lo tenía dicha sobrecarga. Sin embargo Orós, E. (2015) en Lima evidenció que del $100 \%$ (29) de los profesionales el 80\% (23) tuvieron burnout de nivel medio, $17 \%(05)$ bajo y $3 \%(01)$ alto; mientras que Montenegro (2013) en su estudio 
encontró que en Moyobamba y en Tarapoto en un $90 \%$ y $91.2 \%$ respectivamente no presentaban este síndrome.

El Síndrome de burnout representa una verdadera amenaza no sólo para la salud del personal de salud sino también para la atención que brinda a los pacientes ya que al padecer dicho síndrome se pierden la sensibilidad y el humanismo lo cual genera distanciamiento afectivo.

También repercute dentro de la institución ya que genera un aumento en el ausentismo, apatía hacia la organización, aislamiento y déficit de la calidad del trabajo. También opaca el reconocimiento social de estos profesionales, razón por la cual es importante implementar acciones y programas que contribuyan a disminuir su aparición o su exacerbación. Cabe resaltar intervenciones para mejorar la calidad de la asistencia sanitaria y las condiciones de trabajo dentro de las instituciones; toda vez que es importante revelar el arte del cuidado y esto implica el cuidado de si y el cuidado del otro (persona cuidada, profesionales que cuidan y el cuidado de la institución).

En el hospital de Jaén los profesionales de enfermería en un $83.3 \%$ (50) presenta un regular clima Organizacional y el $11.7 \%$ (7) presenta un Clima Organizacional Malo. Un buen clima organizacional genera una institución servidora saludable para lograr un desempeño profesional excelente traducido en salud integra y productividad alta; que facilita el logro de la misión y visión de la institución donde labora tornándose en calidad de un servicio (Tabla 3 ).

Existe similitudes con las investigaciones de Arboleda, L. (2016) Callao cuando concluye que el $58.6 \%$ percibe un clima laboral regular y solo un $27.6 \%$ lo percibe bueno así como también, Arque, I. (2017) en Lima cuando obtuvo que el 73.3\% presenta un clima organizacional de nivel regular y un $20 \%$ bueno.

Un clima laboral armonioso es cuando los trabajadores estén íntimamente relacionado con su trabajo y a los beneficios del mismo, propicia que la persona evidencie un desempeño y una actitud favorable y eficiente a su trabajo, creando así nuevas oportunidades para gerenciar actitudes positivas hacia su empleo, de lo contrario, el trabajador estará expuesto a presentar posibles alteraciones en su personalidad y cambios bruscos de interés hacia el trabajo y su entorno.

En este estudio se ha encontrado que más del $50 \%$ de los profesionales de enfermería de esta institución presentan cansancio emocional y despersonalización en un nivel alto aun así se siente realizados. (Tabla
4).

Arias, L. \& Muñoz, A. (2016) en Arequipa evidencia que el $21,3 \%$ de enfermeras presentó niveles severos de agotamiento emocional y baja realización personal, y que el 29,8 \% tuvo altos niveles de despersonalización y se relacionan positivamente, asimismo Villanueva, R. \& Albaladejo, R. y colaboradores (2014) en Madrid España. en la dimensión cansancio emocional $48.2 \%$ de nivel alto, para despersonalización $48.1 \%$ como nivel alto y finalmente en baja realización personal con $48,9 \%$ de nivel medio esto manifiesta que el personal de enfermería está más despersonalizado y menos realizado.

Por otro lado Trucios, M. (2016) Huancavelica en sus resultados evidencian que en la dimensión agotamiento emocional el $62.9 \%$ es bajo, en la dimensión de despersonalización el $42.9 \%$ (15) es alto y en la dimensión baja realización personal el $54.3 \%$ es bajo. También Salillas, R. (2017) México en sus resultados evidencian que en la dimensión cansancio emocional llega al $83.3 \%$ en un nivel bajo mientras que en despersonalización y falta de realización personal llegaron a un $45,8 \%$ y $23,6 \%$ de nivel bajo.

Dentro del equipo multidisciplinario de las instituciones de salud, la enfermería es considerada una profesión que requiere el despliegue de actividades que involucran control mental y emocional, pues implica una exposición a diferentes situaciones donde se debe preservar la vida, el cuidado al individuo, la familia y la comunidad; basándose en conocimientos científicos, técnicos y humanísticos, aplicándolos en los tres niveles de atención. Es por ello que dichos profesionales deben estar preparados para superar todo tipo de obstáculos, sin que esto se convierta en algo negativo para su salud y bienestar (Tabla 4).

Respecto a las dimanaciones del clima organizacional, se observa que del el 76.7\% (46), en la dimensión de sistema individual se observa un nivel regular, en la dimensión sistema interpersonal el $66.7 \%$ y en la dimensión sistema organizacional el $56.7 \%$ presenta un nivel regular. El clima organizacional constituye uno de los factores determinantes de los procesos organizativos de gestión, cambio e innovación de una institución, en este caso del nosocomio o centro hospitalario de Jaén. (Tabla 5).

Mantener niveles altos de satisfacción laboral permite mejorar procesos, fomentar el trabajo en equipo, aumentar la calidad y calidez de atención de enfermería y el rendimiento de su productividad, así 
como la satisfacción de los usuarios. Según Alvan, P. \& Arteaga, G. (2013) en Trujillo, mencionan que las enfermeras viven en un clima organizacional de nivel regular.

Los motivos que explicarían esta realidad serían las condiciones laborales no tan buena para el desempeño laboral ya que las características físicas e infraestructura de los servicio de salud, debería ser más amplia y confortable por la cantidad de pacientes a los que se puede atender dentro de cada establecimiento, trabajar en un ambiente con deficiente iluminación, escasa ventilación, y temperatura poco ideal responden a estos resultados, la distribución adecuada del personal que pueda abastecer la atención contando como mínimo con dos enfermeros y su personal técnico en un turno de 6 horas y esto amerita gestionar presupuesto para mejorar la infraestructura, recursos humanos, una reingeniería a los procesos con el único propósito de facilitar y alivianar el trabajo del personal de salud y así evitar posibles problemas hacia la organización y a la del propio profesional que a diario está expuesto a situaciones difíciles que exponen grandemente su salud mental, como física.

El sistema de Salud en este contexto requiere plantear políticas de estado acorde a la realidad en el mundo en que se vive enmarcado en el objetivo del milenio el acceso a los servicios de calidad y eso se va lograr cuando se cuenta con instituciones de salud renovadas donde se considere el trabajo del enfermero y enfermera peruana como un capital humano y social motivado y esmerado en el trabajo que realiza y que demuestren el compromiso institucional de brindar un servicio de calidad al usuario que lo solicitan a gritos en el día a día; esto se visibilizará en una imagen y representación social distinguida en la sociedad.

\section{CONCLUSIONES}

1. El síndrome de burnout tiene una relación altamente significativa con el clima organizacional en profesionales de enfermería del Hospital de Jaén 2018 .

2. Gran parte de las profesiones de enfermería de este hospital presentan síndrome de burnout en niveles considerables.

·3. En su mayoría los profesionales de enfermería de este hospital, dentro de su entorno laboral perciben un regular clima organizacional.

4. Según las dimensiones del síndrome de burnout, se puede evidenciar que en gran parte de los profesionales de enfermería, existe un nivel alto en la dimensión de cansancio emocional y despersonalización, mientras que por otro lado en la dimensión falta de realización personal se encuentra con un nivel bajo.

5. Según dimensiones del clima organizacional, se puede evidenciar que tanto en la dimensión sistema individual, interpersonal y organizacional se encuentra en nivel regular, deduciéndose que el clima dentro de la organización laboral no esta buena.

\section{REFERENCIAS BIBLIOGRÁFICAS}

Acevedo Figueroa, L. (2014). Sindrome de Burnout en Profesional de Enfermeria. Para obtención del Grado de especialista Ciencias de Enfermería, Queretano Mexico.

Albadajedo, R., Villanueva, R., Ortega, P., Astacio , P., Calle, M., \& Dominguez, V. (2004). Síndrome de Burnout en el personal de Enfermería de un Hospital de Madrid. Revista Española de Salud Publica.Alvan, P. \& Arteaga, G. (2013). Clima Organizacional y Calidad de Vida Laboral en Enfermeras del Servicio de Medicina del Hospital Docente. Trujillo, Perú.

Arias, W., \& Muñoz, A. (2016). Síndrome de Burnout en Profesionales de Enfermeria de Arequipa. Revista Cubana de Salud Pública.

Arboléda, L. (2016). Clima organizacional en la satisfacción laboral del personal de enfermería del servicio de medicina. Para optar el Grado de Maestro en Gestión de los Servicios de Salud, Hospital Alberto Sabgal Sologuren, Callao.

Arrque, I. (2017). Percepción de la enfermera sobre el clima organizacional en Centro Quirúrgico. Para optar Título de Especialista en enfermería en Centro Quirurgico, Hospital San Juan de Lurigancho, Lima.

Cañadas , G., García, A., De la Fuente , E., Consepción, L., Gómez, J., \& Cañadas, G. (16 de 08 de 2016). Síndrome de Burnout en profesionales de Enfermería que realizan jornada Física complementaria en es servicios de cuidados críticos. Revista Española de Salud Pública, 90, 1-2.

Camasi, K. (2018). Relación entre el Síndrome de Burnout y Clima Laboral de las Organizaciones: Revisión Conceptual. Para la Obtención de Grado de Bachiller en Psicología, Lima.

Corbalán, V. (01 de 11 de 2017). Clima organizacional de enfermería en los hospitales regionales del Instituto de 
Previsión Social. Mem. Inst. Investig. Cienc. Salud, 11, 41 -54.

Chuco, Y., \& Peña, M. (2018). Clima laboral y sindrome de burnout del profesional de enfermería en emergencia. Para optar el Título Profesional de Licenciado en Enfermería, Hospital Daniel Alcides Carrión, Huancayo.

Gil-Monte, P. (2014). El síndrome de quemarse por el trabajo (sindrome de burnout): aproximaciones Teoricas para su Expliación y Recomendaciones para su Intervención. $\mathrm{R}$ e vi s t a PsicologicaCientífica.com, 69-77. Recuperado el 05 de 07 de 2018

Gómez, M.A y Vicario, O.M. (2008) Clima organizacional: Conceptualización y propuesta de una escala. México: UNAM. $\mathrm{O}$ b t e $\mathrm{n}$ i d o e $n$ : http://www.cienciaytrabajo.cl/cytqa/Pagina s/Desarrollo-y-validaci\%C3\%B3n-de-laEscala-Multidimensional-de-ClimaOrganizacional-(EMCO)-un-estudioemp $\% \mathrm{C} 3 \%$ ADrico-con-profesionales.aspx

Hernandez, R. \& Fernandez, C., \& Baptista, M. (2010). Metodología de la Investigación (Vol. Quinta Edicion ). Mexico: MC Graw Hill.1.

Maslach, C. (1981). Cuestionario Maslach Inventory MBI Rangos de Medida.

Medina, L. (2015). El Sindrome de Burnot en Enfermeras de la Unidad de Cuidados Intensivos de los Hospitales Públicos de la comunidad Autónoma de Madrid. MadridEspaña.

Miravalles, J. 2014 (05 de 07 de 2018). Maslach Burnot Inventoy. Obtenido de Gabinete Psicologico: www.javiermiravalles.es

Montenegro, A. (2013). Síndrome de Burnout en Profesionales de Enfermería del Hospital II1 Moyobamba y Hospital II-2 Tarapoto. San Martín. Moyobamba-Tarapoto: Universidad Nacional Toribio Rodriguez de Mendoza de Amazonas.

Organización Mundial de la Salud. (06 de 11 de 2019). La OMS Reconoce como Enfermedad al Burnout o "Síndromde de estar Quemado". Médicos y Pacientes . Com, 1 2.

Orós, E. (2015). Nivel del Síndrome de Burnout en el personal de enfermería de la UCIP . |Para optar el Título de Especialista en Enfermería Intensivista, Hospital Nacional Alberto Sabogal Sologuren, Lima.

Piñera, M., Rodriguez, A., \& Acosta, S. (marzo de 2016). Clima Organizacional: Percepción de Enfermeras del Nivel Primario de Atención. Revista Cubana de Enfermería. $\mathrm{O}$ b t e $\mathrm{n} \mathrm{i} \mathrm{d} \mathrm{o} \mathrm{d} \mathrm{e}$ http://www.revenfermeria.sld.cu/index.php/ enf/article/view/967/187

Rodríguez, A. (2016). Síndrome de Burnout en el Profesional de Enfermería en Áreas Críticas del Hospital de Tercer Nivel Mexico. Mexico.

Romero, H., Muñoz , Y., Osorio, D., \& Robles, E. (2014). Sindrome de Burnout en el Profesional de Enfermería de un Hospital de Referencia Ibagué. Enfermería Global. Colombia, Ibagué, Colombia.

Salillas, R. (05 de 12 de 2017). Síndrome de Burnout en el profesional de Enfermería en el ámbito Hospitalario. Revista de Enfermería del Trabajo, 65 - 69.

Saucedo, G. (2015). Factores de Risgos Psicosociales del Sindrome de Burnout del Personal de Enfermería del Hospital Regional Virgen de Fátima. Tesis para Optar el Grado de Licenciada en Enfermería , Amazonas, Chachapoyas.

Soliz, Z., Zamudio, 1., Matzumura, J., \& Gutiérrez, H. (16 de 06 de 2016). Relación entre el Clima Organizacional y el Síndrome de

Trucios , M. (2016). Síndrome de burnout en personal de enfermería de centro quirúrgico. Para obtener especialidad en Centro Quirurugico, Hospital Regional Zacarías Correa Valdivia de Huancavelica, Huancavelica. 